\title{
Synthesis and Physicochemical Properties of Polyacrylates and Polymethacrylates with Tetraphenylporphinatocopper(II) and -vanadyl(II)
}

\author{
Nobuyuki Watanabe, Xien Su Cheng, Akira Harada, \\ Mikiharu KAMACHI, ${ }^{\dagger}$ Hirokazu NAKAYAMA, ${ }^{*}$ Wasuke MORI, ${ }^{*}$ \\ and Michihiko KISHITA* \\ Department of Macromolecular Science, Faculty of Science, Osaka University, \\ Toyonaka, Osaka 560, Japan \\ * Depertment of Chemistry, College of General Education, Osaka University, \\ Toyonaka, Osaka 560, Japan
}

(Received January 4, 1989)

\begin{abstract}
Polymers of acrylates and methacrylates containing porphinatoparamagnetic metal ions in their side chains were obtained by the radical polymerization of 5-[4(acryloyloxy)phenyl]-10,15,20-triphenylporphinatoparamagnetic metal ions (AOTPPM) and 5-[4(methacryloyloxy)phenyl]-10,15,20-triphenylporphinatoparamagnetic metal ions (MAOTPPM) $(\mathrm{M}=\mathrm{Cu}(\mathrm{II})$ or $\mathrm{VO}(\mathrm{II}))$ by using $\mathrm{AIBN}$ at $60^{\circ} \mathrm{C}$. The visible spectra of polyAOTPPM and polyMAOTPPM showed hypochromism at the Soret bands and the spectrum of polyAOTPPM showed hyperchromism around $650 \mathrm{~nm}$. These results indicate that the porphyrin moieties bound to the polymer chain are forced to interact electronically each other because of the restriction of their mobility. Their ESR spectra showed that exchange interactions between unpaired electrons on metal ions take place, and there are antiferromagnetic interactions between $\mathrm{VO}$ (II) ions in polyAOTPPVO(II). Much weaker antiferromagnetic interaction was observed between $\mathrm{Cu}(\mathrm{II})$ ions in polyAOTPPCu(II).

KEY WORDS Paramagnetic Species / Radical Polymerization Hypochromism / Exchange Interaction / Antiferromagnetic Interaction / Ferromagnetic Interaction/
\end{abstract}

Polymers containing paramagnetic species in their side chains are considered to be new magnetic materials, because a magnetic ordering of the paramagnetic species through an exchange interaction of unpaired electrons is possible in the polymers. ${ }^{1-7}$

Homopolymerizations of vinyl compounds containing porphyrin moieties have not been reported, although polymers containing porphyrin moieties have been prepared by polymer reactions and copolymerizations. We reported previously the radical polymerizations of 5-[4-(acryloyloxy)phenyl]-10,15,20-triphenylporphyrin (AOTPP) and 5-[4-(methacryloyloxy)phenyl]-10,15,20-triphenylpor- phyrin (MAOTPP), ${ }^{8.9}$ and additionally, that polyacrylate containing porphinatosilver (polyAOTPPAg(II)) indicated the occurrence of antiferromagnetic interaction between unpaired electrons in the side chains. ${ }^{3}$ Recently, we also succeeded in the preparation of a polymer containing metalloporphyrins by the radical polymerization of the corresponding monomers.

In this paper, we report the radical polymerization of 5-[4-(acryloyloxy)phenyl]-10,15,20triphenylporphinatoparamagnetic metal ions (AOTPPM: $\mathrm{M}=\mathrm{Cu}(\mathrm{II})$ and $\mathrm{VO}(\mathrm{II})$ ) and 5-[4(methacryloyloxy)phenyl]-10,15,20-triphenylporphinatoparamagnetic metal ions (MAO- 
TPPM: $\quad M=\mathrm{Cu}(\mathrm{II})$ and $\mathrm{VO}(\mathrm{II})$ ) and 5-[4(methacryloyloxy)phenyl]-10,15,20-triphenylporphinatoparamagnetic metal ions (MAOTPPM: $\mathrm{M}=\mathrm{Cu}(\mathrm{II})$ and $\mathrm{VO}(\mathrm{II})$ ), and 5-[4chemical properties of these polymers.

\section{EXPERIMENTAL}

\section{Materials}

Solvents were purified by distillation under nitrogen. 5-(4-Hydroxyphenyl)-10,15,20-triphenylporphyrin (HOTPP) was prepared from benzaldehyde, $p$-hydroxybenzaldehyde and pyrrole according to the method of Little et al. ${ }^{10}$ (yield $4.3 \%$ ). Acryloyl chloride was prepared from acrylic acid and benzoyl chloride as described previously. ${ }^{11}$ Methacryloyl chloride was prepared from methacrylic acid and benzoyl chloride in a similar way.

5-(4-Hyxdroxyphenyl)-10,15,20-triphenylporphinatocopper(II) (HOTPPCu(II)) was quantitatively obtained by refluxing a solution of $5 \mathrm{~g}(8.0 \mathrm{mmol})$ of HOTPP and $3.2 \mathrm{~g}(16.0$ $\mathrm{mmol}$ ) of copper(II) acetate in $1000 \mathrm{ml}$ chloroform for $1 \mathrm{~h}$. HOTPPVO(II) was prepared by refluxing a solution of $4.7 \mathrm{~g}(7.5 \mathrm{mmol})$ of HOTPP and $11.8 \mathrm{~g}$ of $\mathrm{VOSO}_{4} \cdot \mathrm{H}_{2} \mathrm{O}$ in a mixed solvent of $1500 \mathrm{ml}$ of $\mathrm{N}, \mathrm{N}$-dimethylformamide (DMF), $150 \mathrm{ml}$ of acetic acid, and $50 \mathrm{ml}$ of trichloroacetic acid for $3 \mathrm{~h}$. A crude product was obtained by removal of the solvents, dissolved in chloroform, and chromatographed on a silica gel column $(20 \mathrm{~cm}$ length and $5 \mathrm{~cm}$ diameter) using chloroform as the eluent. HOTPPVO(II) was isolated by removal of the solvent from the second fraction, dissolved in chloroform and purified by reprecipitation from $n$-hexane.

AOTPPCu(II) and MAOTPPCu(II) were prepared as follows: HOTPPCu(II) $(3.0 \mathrm{~g}, 4.3$ mmol) and triethylamine $(15 \mathrm{ml}, 108 \mathrm{mmol})$ were dissolved in $500 \mathrm{ml}$ of tetrahydrofuran (THF). The solution was cooled to ice temperature and acryloyl chloride $(7 \mathrm{ml}, 87 \mathrm{mmol})$ or methacryloyl chloride $(8.5 \mathrm{ml}, 87 \mathrm{mmol})$ was added dropwise with stirring over a period of $20 \mathrm{~min}$. The reaction mixtures were stirred at room temperature for $30 \mathrm{~min}$. Removal of the solvent afforded a crude product which was dissolved in chloroform and chromatographed on a silica gel $(20 \mathrm{~cm}$ length and $5 \mathrm{~cm}$ diameter). AOTPPCu(II) and MAOTPPCu(II) were isolated by removal of the solvent from the first fraction, and dried at $160^{\circ} \mathrm{C}$ for $24 \mathrm{~h}$ under reduced pressure. AOTPPCu(II) and MAOTPPCu(II) were purified by repeated reprecipitation from chloroform and methanol. Yields $2.5 \mathrm{~g}(77 \%)$ and $2.7 \mathrm{~g}(83 \%)$, respectively.

AOTPPCu(II); IR (KBr) 1747 (C=O), 1633 $(\mathrm{C}=\mathrm{C}), 900 \mathrm{~cm}^{-1}(\mathrm{C}=\mathrm{C}-\mathrm{H})$; Anal. Calcd for $\mathrm{C}_{47} \mathrm{H}_{30} \mathrm{~N}_{4} \mathrm{O}_{2} \mathrm{Cu}: \mathrm{C}, 75.64 \% ; \mathrm{H}, 4.05 \% ; \mathrm{N}$, $7.51 \%$; Cu, 8.5\%. Found: C, $75.32 \%$; $\mathrm{H}$, $4.30 \%$;, $7.47 \%$; Cu, $8.5 \%$.

MAOTPPCu(II); IR (KBr) $1742(\mathrm{C}=\mathrm{O})$, $1636(\mathrm{C}=\mathrm{C}), 945 \mathrm{~cm}^{-1} \quad(\mathrm{C}=\mathrm{C}-\mathrm{H}) ;$ Anal. Calcd for $\mathrm{C}_{48} \mathrm{H}_{32} \mathrm{~N}_{4} \mathrm{O}_{2} \mathrm{Cu}: \mathrm{C}, 75.82 \% ; \mathrm{H}$, $4.24 \%$; N, 7.37\%; Cu, 8.4\%. Found: C, $75.68 \%$; $\mathrm{H}, 4.29 \%$;, $7.32 \%$; Cu, $8.6 \%$.

AOTPPVO(II) and MAOTPPVO(II) were obtained (at $61 \%$ and $69 \%$, respectively) by reactions of HOTPPVO(II) $(1.7 \mathrm{~g}, 2.4 \mathrm{mmol})$ with acryloyl chloride $(4.0 \mathrm{ml}, 49 \mathrm{mmol})$ and with methacryloyl chloride $(4.8 \mathrm{ml}, 49 \mathrm{mmol})$ in the presence of $200 \mathrm{ml}$ of chloroform containing triethylamine $(8.5 \mathrm{ml}, 61 \mathrm{mmol})$.

AOTPPVO(II); IR (KBr) $1748 \quad(\mathrm{C}=\mathrm{O})$, $1630(\mathrm{C}=\mathrm{C}), 900 \mathrm{~cm}^{-1} \quad(\mathrm{C}=\mathrm{C}-\mathrm{H}) ;$ Anal. Calcd for $\mathrm{C}_{47} \mathrm{H}_{30} \mathrm{~N}_{4} \mathrm{O}_{3} \mathrm{~V}$ : C, $75.30 \% ; \mathrm{H}$, $4.03 \%$; N, $7.47 \%$; V, 6.8\%. Found: C, $75.77 \%$; $\mathrm{H}, 4.12 \%$; N, $7.50 \%$; V, $6.4 \%$.

MAOTPPVO(II); IR (KBr) $1740(\mathrm{C}=\mathrm{O})$, $1635(\mathrm{C}=\mathrm{C}), 947 \mathrm{~cm}^{-1} \quad(\mathrm{C}=\mathrm{C}-\mathrm{H})$; Anal. Calcd for $\mathrm{C}_{48} \mathrm{H}_{32} \mathrm{~N}_{4} \mathrm{O}_{3} \mathrm{~V}$ : C, $75.49 \% ; \mathrm{H}$, $4.22 \%$; N , 7.34\% ; V $6.7 \%$. Found: C, $75.79 \%$; $\mathrm{H}, 4.40 \%$; N , $7.36 \%$; V, $6.1 \%$.

Radical polymerizations of these monomers were performed with 2,2'-azobis(isobutyronitrile) (AIBN) at $60^{\circ} \mathrm{C}$ using benzonitrile as a solvent. PolyAOTPPM and polyMAOTPPM (M: $\mathrm{Cu}(\mathrm{II})$ or $\mathrm{VO}(\mathrm{II})$ ) were prepared as follows: $0.7 \mathrm{~g}$ of AOTPPM or MAOTPPM and 
$1.5 \mathrm{mg}$ of AIBN were put into an ampoule, and then dissolved in $1.7 \mathrm{ml}$ of benzonitrile. The solution was degassed, sealed under high vacuum, and allowed to stand at $60^{\circ} \mathrm{C}$ for $50 \mathrm{~h}$. In $\mathrm{M}=\mathrm{Cu}(\mathrm{II})$, the polymers were purified by three dissolution-precipitation-separation steps from chloroform-hexane. In $\mathbf{M}=$ VO(II), the polymers were purified by three dissolution-precipitation-separation steps from chloroform-methanol. Elemental analyses of the obtained polymers are as follows: PolyAOTPPCu: C, $74.81 \% ; \mathrm{H}, 4.12 \% ; \mathrm{N}$, $7.48 \%$; $\mathrm{Cu}, 8.4 \%$, and PolyAOTPPVO: C, $75.15 \%$; H, $4.12 \%$; N, $7.51 \%$; V, 6.1\%. These values are consistent with the results of the corresponding monomers, indicating that addition polymerizations took place.

Molecular weights of the polymers were determined by GPC measurements using THF as a solvent. The molecular weights were calibrated by standard polystyrenes.

\section{Measurements}

UV-visible spectra were measured in benzene with a Shimadzu UV-visible recording spectrophotometer UV-2100. IR spectra were recorded in $\mathrm{KBr}$ disks with a JASCO infrared spectrophotometer DS-402G. GPC analysis was carried out with a Toyo Soda high-speed liquid chromatograph HGC-810A. Thermogravimetric analyses were performed under air using a Rigaku thermoanalyser TG-DTA at a heating rate of $20^{\circ} \mathrm{C} \mathrm{min}^{-1}$. Gram magnetic susceptibility $\left(\chi_{\mathrm{g}}\right)$ was determined by the Gouy method at room temperature using distilled water $\left(\chi_{\mathrm{g}}=-0.72 \times 10^{-6} \mathrm{cgsemu}\right)$ as the standard. The contribution of atmosphere to the magnetic susceptibility was corrected by adding $0.03 \times 10^{-6}$ cgsemu to each value of $\chi_{\mathrm{g}}$. The temperature dependence of $\chi_{\mathrm{g}}$ was determined by the Faraday method, using a Cahn 1000 electric balance in a temperature range from $4 \mathrm{~K}$ to $300 \mathrm{~K}$. The molar magnetic susceptibility $\left(\chi_{\mathrm{A}}\right)$ was corrected by the following equation to eliminate diamagnetic contribution from the ligand.

$$
\chi_{\mathrm{A}}=\chi_{\mathrm{g}} \cdot M-\chi_{\mathrm{dia}}
$$

where $M$ is the molecular weight of the monomer unit of the polymer, and the value of $\chi_{\text {dia }}$ for polyAOTPP was calculated to be $-505 \times 10^{-6}$ cgsemu using the Pascal constant. ${ }^{12}$ ESR spectra were recorded with a JEOL Model JES-FE1X ESR spectrometer with a $100 \mathrm{kHz}$ modulator. The $g$-values were estimated using $\mathrm{MnO}$, commercially obtained from JEOL, as the standard sample.

\section{RESULTS AND DISCUSSION}

\section{Radical Polymerization}

The results of the radical polymerizations of AOTPPM and MAOTPPM (M: $\mathrm{Cu}(\mathrm{II})$ or $\mathrm{VO}(\mathrm{II})$ ) are shown in Table I. The polymers obtained were soluble in benzene, benzonitrile, THF, DMF, and chloroform, and insoluble in methanol, hexane, acetone, and water.

The IR spectra of AOTPPCu(II) and polyAOTPPCu(II) are shown in Figure 1. The IR bands of the monomer at 1633 and 1747 $\mathrm{cm}^{-1}$ were assignable to absorptions due to olefinic and carbonyl bonds of unsaturated ester, respectively. In polyAOTPPCu(II), the former disappeared and the latter shifted to $1760 \mathrm{~cm}^{-1}$, indicating that the carbonyl bond of the unsaturated ester changed to that of saturated ester. Absorption bands characteristic of the porphyrin ring about $700-800 \mathrm{~cm}^{-1}$ remained unchanged after polymerization. Similar change was observed on comparing

Table I. Radical polymerizations of AOTPPM and MAOTPPM ${ }^{\mathrm{a}}$

\begin{tabular}{lcc}
\hline & \multicolumn{2}{c}{ Yield } \\
\cline { 2 - 3 } Monomer & $\%$ & Molwt \\
& $\%$ & \\
\hline AOTPPCu(II) & 54 & 108000 \\
MAOTPPCu(II) & 71 & 59000 \\
AOTPPVO(II) & 47 & 22000 \\
MAOTPPVO(II) & 44 & 19000 \\
\hline${ }^{a}[$ Monomer] $=0.5 \mathrm{M} ;$ & {$[\mathrm{AIBN}]=5 \mathrm{mM} ;$} & solvent, \\
benzonitrile. & &
\end{tabular}


the IR spectra of AOTPPVO(II) and polyAOTPPVO(II). These findings show that polymerization takes place through the $\mathrm{C}=\mathrm{C}$ bond of the monomers.

The visible spectra of AOTPPCu(II) and polyAOTPPCu(II) are shown in Figure 2. AOTPPCu(II) had a Soret band at $414 \mathrm{~nm}$ and $\mathrm{Q}$ bands at 539 and $575 \mathrm{~nm}$, while poly-

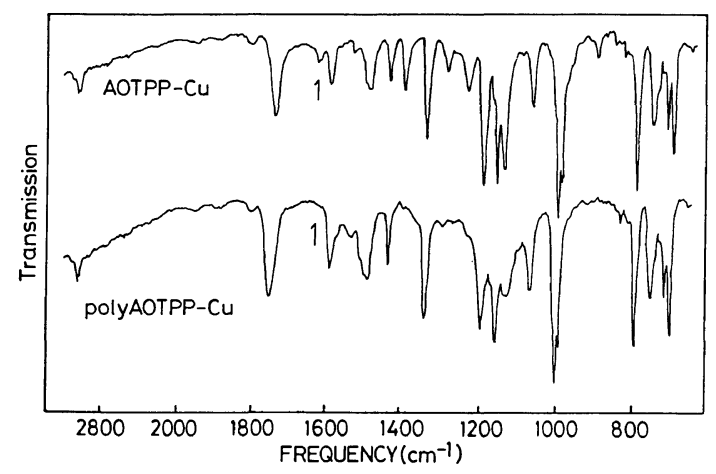

Figure 1. IR spectra of AOTPPCu(II) and polyAOTPPCu(II).
AOTPPCu(II) had a Soret band at $413 \mathrm{~nm}$ and $Q$ bands at 540 and $575 \mathrm{~nm}$, and a new absorption band at $619 \mathrm{~nm}$. The Soret band of polyAOTPPCu(II) was much weaker and broader than that of the monomer, although the concentration of the TPP moieties of polyAOTPPCu(II) was equal to that of the monomer. Similar change in absorption bands was observed on comparing the visible spectra of AOTPPVO(II) and polyAOTPPVO(II). The visible spectra of MAOTPPCu(II) and polyMAOTPPCu(II) are shown in Figure 3. A decrease in the value of $\varepsilon$ of the Soret band of polyMAOTPPCu(II) was also observed. However, the $\mathrm{Q}$ bands of the polymer were almost the same as those of MAOTPPCu(II), and no new absorption band was observed. Similar difference of visible spectra was observed on comparing the MAOTPPVO(II) and polyMAOTPPVO(II). A decrease in $\varepsilon$ of the Soret band of the polymer and appearance of a new absorption band at $619 \mathrm{~nm}$ were probably

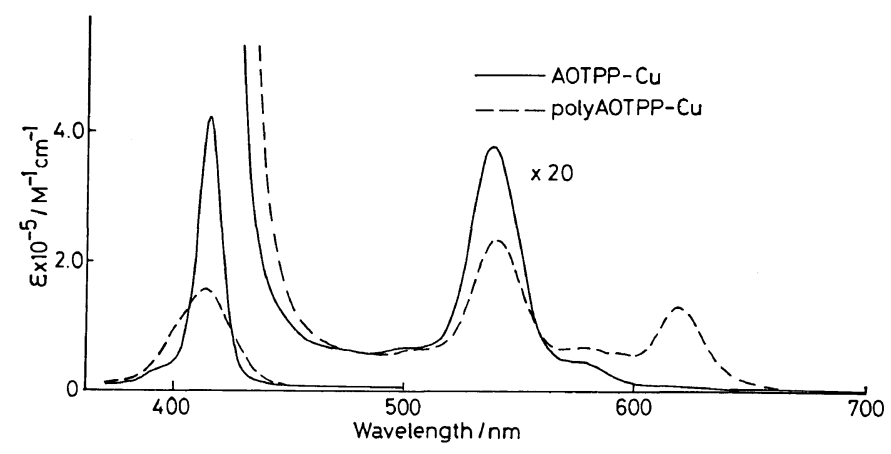

Figure 2. Visible spectra of AOTPPCu(II) and polyAOTPPCu(II) in benezene.

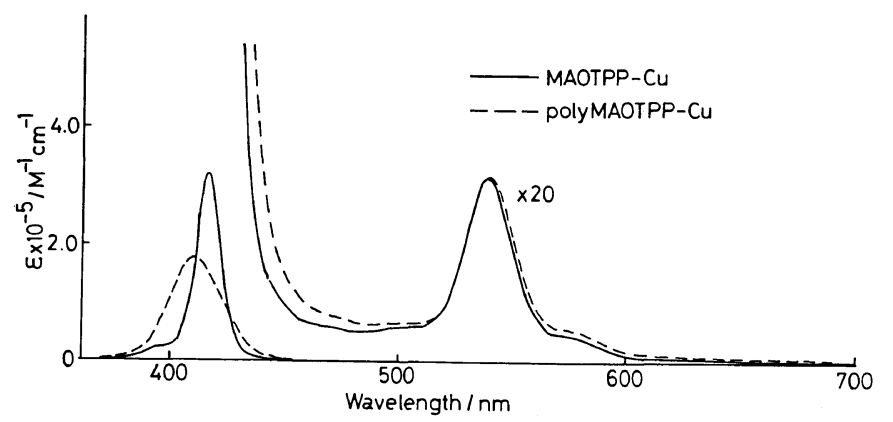

Figure 3. Visible spectra of MAOTPPCu(II) and polyMAOTPPCu (II) in benezene. 


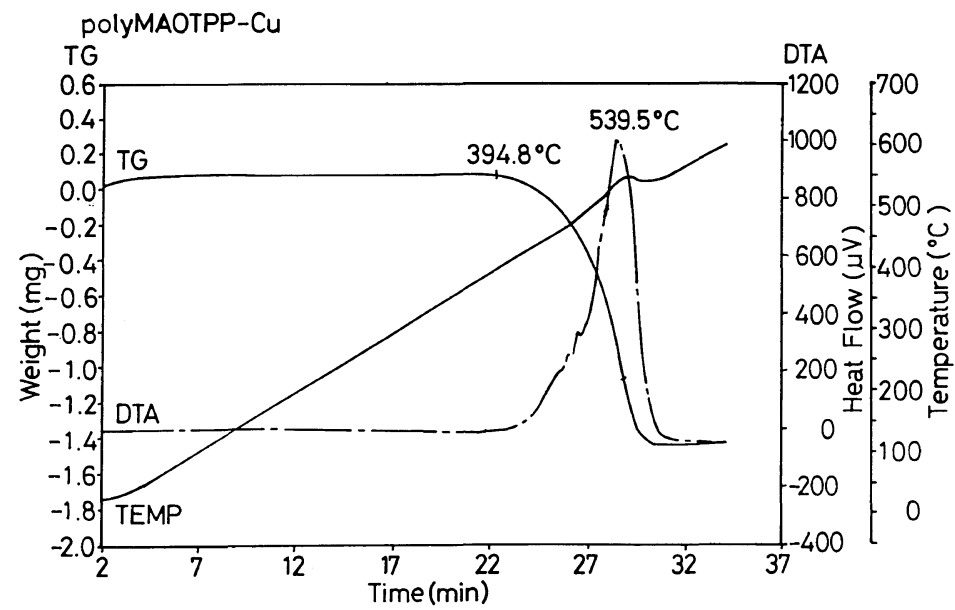

Figure 4. Thermal analysis of polyMAOTPPCu(II): Weight of sample used, $1.8 \mathrm{mg}$; heating rate, $20^{\circ} \mathrm{Cmin}^{-1}$.

caused either by electronic interactions between the porphyrin moieties or by the formation of chlorin moieties from the porphyrin moieties. ${ }^{13}$ Since no new absorption bands at $619 \mathrm{~nm}$ were observed in polyMAOTPPCu(II) and polyMAOTPPVO(II), chlorin was not formed during the radical polymerization of AOTPPCu(II) and AOTPPVO(II). Accordingly, decrease in the values at the Soret bands and the appearance of new absorption bands at $619 \mathrm{~nm}$ in polyAOTPPCu(II) and polyAOTPPVO(II) are ascribable to the electronic interaction between the porphyrin moieties, because these bulky moieties bound to polyacrylates can make stronger electronic interactions due to their proximity than those to polymethacrylates. ${ }^{9}$ The results of visible spectra indicate that the methyl group bound to the polymer chain causes the conformational change of the polymer chain, so that the interactions between the side chains are stronger in polyacrylates than in polymethacrylates.

\section{Heat Stability}

The thermal behavior of polyAOTPPM and polyMAOTPPM was investigated by thermogravimetric analysis. A typical example
Table II. Temperatures for the decomposition of polyAOTPPM and polyMAOTPPM

\begin{tabular}{lc}
\hline & Decom. temp \\
\cline { 2 - 2 } Polymer & $\mathrm{C}$ \\
\hline PolyAOTPPCu(II) & 381 \\
PolyMAOTPPCu(II) & 394 \\
PolyAOTPPVO(II) & 384 \\
PolyMAOTPPVO(II) & 413 \\
\hline
\end{tabular}

is shown in Figure 4. Decomposition temperatures of polyAOTPPM and polyMAOTPPM are shown in Table II. No weight loss was observed from room temperature to $380^{\circ} \mathrm{C}$ for any polymers. Thermal stabilities of these polymers were higher than those of commercially available polyacrylates and polymethacrylates.

\section{ESR Studies}

The ESR spectra of AOTPPVO(II) and polyAOTPPVO(II) in benzene at room temperature are shown in Figure 5. Isotropic eight lines observed for AOTPPVO(II) were due to the magnetic interactions between an unpaired electron and a vanadium nucleus $(I=7 / 2)$. The spectrum of polyAOTPPVO(II) was broader 


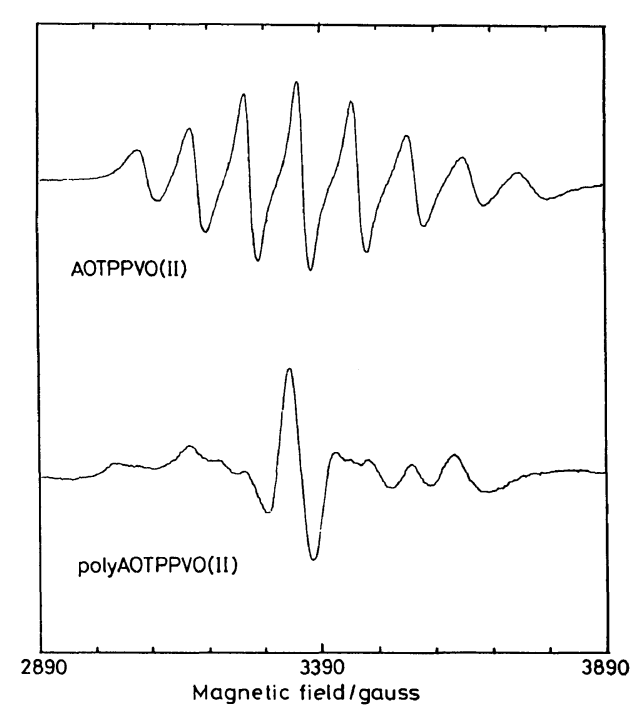

Figure 5. ESR spectra of AOTPPVO(II) and polyAOTPPVO(II) in benezene at room temperature.

than that of AOTPPVO(II), and, in addition, a single line at $g=2.002$ was observed in polyAOTPPVO(II). The difference in ESR spectra between MAOTPPVO(II) and polyMAOTPPVO(II) was almost the same as that between AOTPPVO(II) and polyAOTPPVO(II). Since the ESR spectrum of AOTPPVO(II) did not change on adding the same concentration of polyMMA as that of polyMAOTPPVO(II), the difference in the ESR spectra between monomers and polymers shows that the magnetic interactions of unpaired electrons occur between TPPVO(II) moieties in polyAOTPPVO(II) and polyMAOTPPVO(II).

The ESR spectra of MAOTPPCu(II) and polyMAOTPPCu(II) in benzene at room temperature are shown in Figure 6. Hyperfine structures observed for MAOTPPCu(II) were due to magnetic interactions of an unpaired electron with a copper and four nitrogen nucleus. For polyMAOTPPCu(II), hyperfine structures disappeared and the spectrum became broader. Since the ESR spectrum of MAOTPPCu(II) did not change on adding the same concentration of polyMMA as that of polyMAOTPPCu(II), the broadening of the

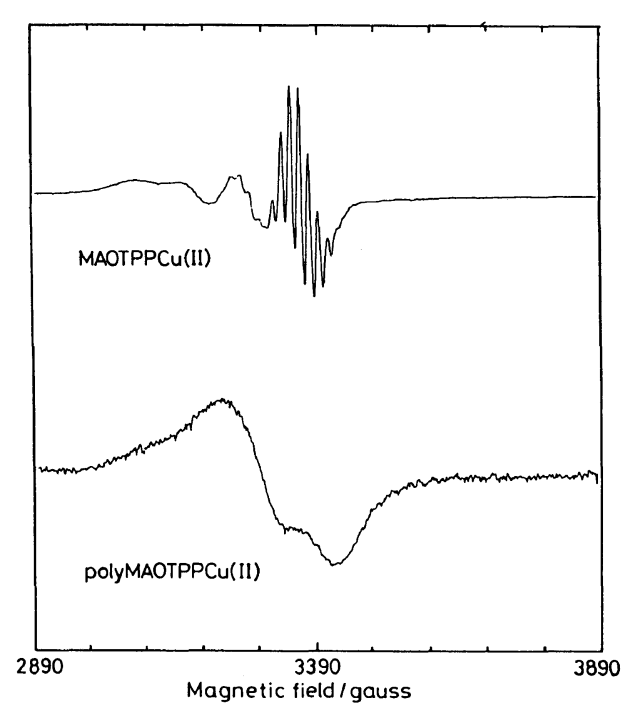

Figure 6. ESR spectra of MAOTPPCu(II) and poly MAOTPPCu(II) in benezene at room temperature.

spectrum of polyMAOTPPVO(II) is ascribable to the exchange interaction of unpaired electrons between copper ions in the polymer.

\section{Magnetic Properties}

The temperature dependence of the effective magnetic moment and the inverse of magnetic susceptibility of polyAOTPPVO(II) (Figure 7) obeys the Curie-Weiss law $\left(\chi_{\mathrm{A}}=C /(T-\theta)\right)$ with $\theta=-125 \pm 45 \mathrm{~K}$ in temperature range from $150 \mathrm{~K}$ to $300 \mathrm{~K}$, which indicates the existence of a relatively strong antiferromagnetic interactions between unpaired electrons in the porphyrin moieties. PolyAOTPPCu(II) obeys the Curie-Weiss law with $\theta=$ $-60 \pm 50 \mathrm{~K}$, indicating that there is a weak antiferromagnetic interaction between unpaired electrons.

The observed difference between polyAOTPPCu(II) and polyAOTPPVO(II) shows that the antiferromagnetic interaction in polyAOTPPCu(II) is weaker than that in polyAOTPPVO(II). This suggests that the oxygen atom of $\mathrm{VO}$ (II) might enhance antiferromagnetic interactions between paramagnetic metal ions by superexchange interactions through 


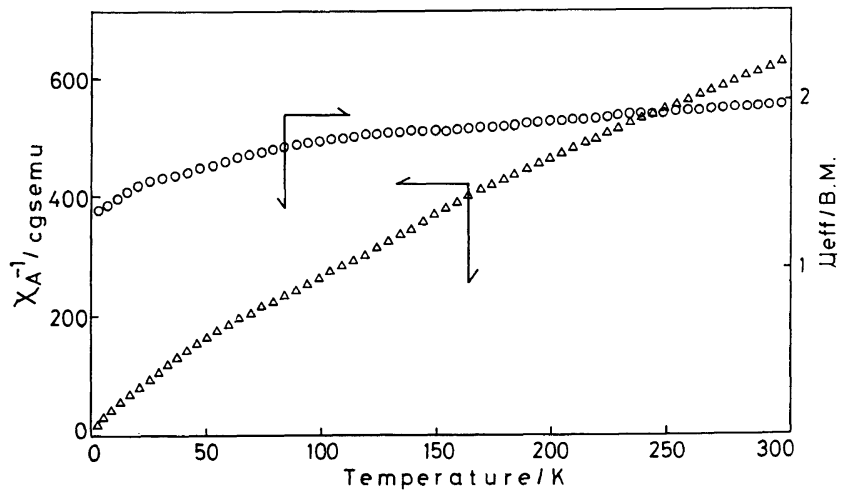

Figure 7. Temperature dependence of the effective magnetic moment and the inverse of magnetic susceptibility of polyAOTPPVO(II).

oxygen atoms. ${ }^{14}$

\section{CONCLUSION}

We succeeded in preparing polyacrylates and polymethacrylates containing tetraphenylporphinatocopper(II) and -vanadyl(II) in their side chains by radical polymerizations of monomers containing these metalloporphyrins. The interactions between porphyrin moieties of the polymers were stronger than those of the corresponding monomers. As magnetic properties of the polymers, we found that antiferromagnetic interactions between unpaired electrons on metal ions take place in polyAOTPPVO(II) and polyAOTPPCu(II); the antiferromagnetic interaction is stronger in polyAOTPPVO(II) than in polyAOTPPCu(II).

Acknowledgment. This work was partly supported by a Grant-in-Aid for Scientific Research or Priority Areas of "Macromolecular Complexes" (No. 63612001) from the Ministry of Education, Science, and Culture of Japan.

\section{REFERENCES}

1. A. A. Ovchinnikov, Dokl. Acad. Nauk., USSR, 236, 928 (1977).

2. A. L. Buchachenko, Dokl. Acad. Nauk., USSR, 244, 1146 (1979).

3. M. Kamachi, H. Akimoto, W. Mori, and M. Kishita, Polym. J., 16, 23 (1984).

4. Y. Pei, M. Verdaguer, O. Kahn, J. Sletten, and J. P. Renard, J. Am. Chem. Soc., 108, 7428 (1986).

5. J. B. Torrance, S. Oostra, and A. Nazzal, Synth. Met., 19, 709 (1987).

6. Y. Korshak, T. V. Medvedeva, A. A. Ovchinnikov, and V. N. Spector, Nature (London), 326, 370 (1987).

7. O. Kahn, Y. Pei, M. Verdaguer, J. P. Renard, and J. Sletten, J. Am. Chem. Soc., 110, 782 (1988).

8. M. Kamachi, H. Akimoto, and S. Nozakura, $J$. Polym. Sci., Polym. Lett. Ed., 21, 693 (1983).

9. M. Kamachi, X. S. Cheng, T. Kida, A. Kajiwara, M. Shibasaka, and S. Nagata, Macromolecules, 20, 2665 (1987).

10. R. G. Little, J. A. Anton, P. A. Loach, and S. A. Ibers, J. Heterocyc. Chem., 12, 343 (1975).

11. M. Kamachi, M. Fujii, S. Ninomiya, S. Katsuki, and S. Nozakura, J. Polym. Sci., Polym. Chem. Ed., 20, 1489 (1980).

12. A. Earnshaw, "Introduction to Magnetochemistry," Academic Press, London, 1968. pp. 4-8.

13. "Porphyrins and Metalloporphyrins," K. M. Smith, Ed., Elsevier, New York, 1975, p 23 and pp 641 643.

14. A. S. Chakravarty, "Introduction to the Magnetic Properties of Solid," John Wiley and Sons, New York, N.Y., 1980, pp. 458-464. 